\title{
RNA-Seq Identifies Key Reproductive Gene Expression Alterations in Response to Cadmium Exposure
}

\author{
Hanyang Hu, Xing Lu, Xiang Cen, Xiaohua Chen, Feng Li, and Shan Zhong \\ Department of Medical Genetics, School of Basic Medical Science, Wuhan University, Wuhan 430071, China \\ Correspondence should be addressed to Shan Zhong; zhongshan@whu.edu.cn
}

Received 14 April 2014; Revised 7 May 2014; Accepted 7 May 2014; Published 27 May 2014

Academic Editor: Leng Han

Copyright (C) 2014 Hanyang Hu et al. This is an open access article distributed under the Creative Commons Attribution License, which permits unrestricted use, distribution, and reproduction in any medium, provided the original work is properly cited.

\begin{abstract}
Cadmium is a common toxicant that is detrimental to many tissues. Although a number of transcriptional signatures have been revealed in different tissues after cadmium treatment, the genes involved in the cadmium caused male reproductive toxicity, and the underlying molecular mechanism remains unclear. Here we observed that the mice treated with different amount of cadmium in their rodent chow for six months exhibited reduced serum testosterone. We then performed RNA-seq to comprehensively investigate the mice testicular transcriptome to further elucidate the mechanism. Our results showed that hundreds of genes expression altered significantly in response to cadmium treatment. In particular, we found several transcriptional signatures closely related to the biological processes of regulation of hormone, gamete generation, and sexual reproduction, respectively. The expression of several testosterone synthetic key enzyme genes, such as Star, Cypllal, and Cyp17al, were inhibited by the cadmium exposure. For better understanding of the cadmium-mediated transcriptional regulatory mechanism of the genes, we computationally analyzed the transcription factors binding sites and the mircoRNAs targets of the differentially expressed genes. Our findings suggest that the reproductive toxicity by cadmium exposure is implicated in multiple layers of deregulation of several biological processes and transcriptional regulation in mice.
\end{abstract}

\section{Introduction}

Cadmium is an environmental and occupational toxic heavy metal that is widely used in industrial process and consumer products. The usual pattern of the nonoccupational cadmium intake is mainly from food, drinking water, and smoking [1], which caused several diseases by toxically targeting the lung, liver, kidney, bone, and the cardiovascular system as well as the immune and the reproductive system [2]. The multiple mechanisms involved in response to cadmium include modulating cell cycle, DNA repair process, DNA methylation status, altering gene expression, and several signaling pathways in carcinogenesis and other diseases [3-5].

It has been well documented that cadmium exposure leads to the impairment of male and female reproductive system both in human and animals. The high level cadmium in the serum and seminal fluid positively correlated to the azoospermia in the infertile Nigerian males [6]. In the female reproductive system, cadmium exposure leads to failure to ovulate, defective steroidogenesis, suppressed oocyte maturation, and failure of developmental progression in preimplantation embryo and implantation. Moreover, by using the established animal model, the cadmium exposure causing the reproductive system damage is associated with a series of abnormalities, including disruption of blood-testis barrier, testicular necrosis, disruption of blood-epididymis barrier, and abnormal sperm morphology [7]. In addition to reproductive system, recent study also indicated that prenatal cadmium exposure perturbs the vascular and immune system of the murine offspring $[8,9]$, implicating the role of cadmium exposure in offspring health. A number of mechanisms of reproductive toxicity of cadmium have been suggested, including ionic and molecular mimicry, interference with cell adhesion and signaling, oxidative stress, genotoxicity, and cell cycle disturbance [7]. Although the DNA microarray was employed to study the transcriptional gene alternation in cell lines and peripheral blood cells exposed to cadmium and identified several differentially expressed genes as well as signaling pathways $[10,11]$, the comprehensive understanding 
of the mechanisms that are responsible for the toxicity of chronic cadmium exposure in testis is still lacking.

In this study, we performed the RNA-seq to profile the alterations of gene expression in response to chronic cadmium exposure. By analyzing the transcriptome between the cadmium treated and untreated mice, we identified a number of transcriptional signatures, which provided mechanistic insight into the mechanism of how the male reproductive system is affected by chronic cadmium exposure.

\section{Materials and Methods}

2.1. Chemicals. Cadmium chloride $\left(\mathrm{CdCl}_{2}\right)$ was purchased from Sigma Chemical Co. (St. Louis, MO).

2.2. Animals and Experimental Design. Thirty six-week-old male Chinese Kun Ming (KM) mouse weighing about 30-32 g were used in the experiment. The animals were obtained from Wuhan University Center for Animal Experiments/A3-Lab. All animals were housed in a laboratory-controlled environment $\left(25^{\circ} \mathrm{C}, 50 \%\right.$ humidity, and light: dark cycle $\left.12 \mathrm{~h}: 12 \mathrm{~h}\right)$. The animals were permitted free access to food and drinking water ad libitum. The food for mice was purchased from Wuhan University Center for Animal Experiment. All animal experiments were carried out in strict accordance with the recommendations in the Regulations for the Administration of Affairs Concerning Experimental Animals of China. The protocol was approved by the Wuhan University Center for Animal Experiment (approved permit number: SCXK 20080004). All surgery was performed under sodium pentobarbital anesthesia, and all efforts were made to minimize suffering.

After acclimatization and one-week observation, we found the daily food consumption per mouse was about 6$8 \mathrm{~g}$. Then all animals were randomly divided equally into three groups and every five mice were housed in a cage. To calculate the consumption of food containing cadmium, we make high-cadmium food as $0.3 \mathrm{mg} \mathrm{CdCl}_{2} / \mathrm{g}$ and lowcadmium food as $0.15 \mathrm{mg} \mathrm{CdCl}_{2} / \mathrm{g}$. Then, each cage of high level cadmium-exposed group was supplied with $50 \mathrm{~g}$ highcadmium food daily, and low level cadmium-exposed group was supplied with $50 \mathrm{~g}$ low-cadmium food as well. On the following day, we collected and removed the remaining food and residue and new $50 \mathrm{~g}$ food was given. By deducting the weights of remaining food and residue, we calculated that the food intake for cadmium treated mice was $6.5 \pm 0.8 \mathrm{~g}$. Therefore, the intake of cadmium in the high group was considered as $1.95 \pm 0.24$ g per mouse daily and in the low group was considered as $0.975 \pm 0.12 \mathrm{~g}$ per mouse daily. Subsequently, all animals were treated with the according food for 6 months, in which the mice of control group were treated with the same amount of food without $\mathrm{CdCl}_{2}$. We chose these specific dose and period of cadmium exposure as our preliminary results showed that cadmium accumulation in serum and testis reached the highest value at six months, and the mice treated with such dose of cadmium did not show abnormalities or other health problems. Then the blood samples of 5 different mice in each group were randomly collected. Serum were separated by centrifugation from the blood samples above and stored at $-80^{\circ} \mathrm{C}$ until assay for the cadmium concentration using the graphite furnace atomic absorption spectrometry (GFAAS) method. Testosterone in serum was measured using Testosterone Parameter Assay kit (R\&D, USA).

\subsection{RNA Isolation and Preparation for Next-Generation} Sequencing. A total of 9 testis samples (three samples from each group) were selected for RNA isolation. Total RNA was isolated using Trizol Reagent (Invitrogen) according to the manufacturer's instructions. Then these RNA samples were sent to Analytical \& Testing Center at Institute of Hydrobiology, Chinese Academy of Sciences (http://www.ihb.ac.cn/ fxcszx/) for the verification of RNA integrity. Then one RNA sample from each group was collected for pair-ends transcriptome sequencing under the Illumina Genome Analyzer IIx platform. The sequencing data have been deposited in NCBI Sequence Read Archive (SRA, http://www.ncbi.nlm .nih.gov/Traces/sra/) with the accession number SRP032958.

\subsection{Read Alignment with TopHat and RNASEQR. Raw} data were mapped to the mouse reference genome ( $\mathrm{mml}$ downloaded from UCSC) using TopHat (version 2.0.3) and RNASEQR (version 1.0.2) software, respectively [12, 13]. Prebuilt genomic indices were created by bowtie and provided to the alignment software for reads mapping. TopHat removes a few low-quality score reads then aligns the reads that are directly mapped to the reference genome. It then determines the possible location of gaps in the alignment based on splice junctions flanking the aligned reads and uses gapped alignments to align the reads that were not aligned by Bowtie in the first step. Compared with other alignment tools, RNASEQR takes advantages of annotated transcripts and genomic reference sequences to obtain high quality mapping result by the three sequential processing steps. It aligns the RNA-seq sequences to a transcriptomic reference firstly, then detects novel exons and identifies novel junctions using an anchor-and-align strategy finally. The output of Tophat can be the input of Cufflinks, while the output of RNASEQR can be converted as the input for Cufflinks using the convert_RNASEQRSAM_to_CufflinkSAM.py script.

2.5. Transcriptome Reconstruction. Aligned reads from TopHat and RNASEQR were assembled by Cufflinks (version 2.0.2), an ab-initio tancscriptome assembler that reconstructs the transcriptome based on RNA-seq reads aligned to the genome with a spliced read aligner. To obtain transcriptome assemblies from the aligned reads, we run Cufflinks with default parameters. After that, normalized expression levels were estimated and reported as FPKM (Fragments Per Kilobase of exon per Million fragments mapped). As several assembled transcripts were obtained from each sample, we used Cuffmerge to assemble them into a comprehensive set of transcripts for further downstream differential expression analysis. 
TABLE 1: Primers used for qPCR validation.

\begin{tabular}{llcc}
\hline Gene & Primer sequence $\left(5^{\prime}-3^{\prime}\right)$ & Target size $(\mathrm{bp})$ & $\mathrm{Tm}\left({ }^{\circ} \mathrm{C}\right)$ \\
\hline \multirow{2}{*}{ Actin, beta } & $\begin{array}{l}\text { Forward: CTGTCGAGTCGCGTCCACCC } \\
\text { Reverse: ACATGCCGGAGCCGTTGTCG }\end{array}$ & 128 & 59 \\
\hline \multirow{2}{*}{ Cyp1lal } & $\begin{array}{l}\text { Forward: AGATCCCTTCCCCTGGTGACAATG } \\
\text { Reverse: CGCATGAGAAGAGTATCGACGCATC }\end{array}$ & 192 & 60 \\
\hline Cyp17al & $\begin{array}{l}\text { Forward: CCAGGACCCAAGTGTGTTCT } \\
\text { Reverse: CCTGATACGAAGCACTTCTCG }\end{array}$ & 250 & 59 \\
\hline Tex15 & $\begin{array}{l}\text { Forward: CAAGAGGCGTCGGTCA } \\
\text { Reverse: TGGCTCCAGGCAGAATG }\end{array}$ & 167 & 59 \\
\hline Adam9 & $\begin{array}{l}\text { Forward: ATTTGAGTGGCACAGAC } \\
\text { Reverse: AGTATTGGGATTTGGAG }\end{array}$ & 194 & 59 \\
\hline Dazl & $\begin{array}{l}\text { Forward: CGCTTAGCAAACTACCTG } \\
\text { Reverse: TTCCCGCCACTGAACAA }\end{array}$ & 147 & 60 \\
\hline
\end{tabular}

2.6. Differential Expression Analysis. In order to determine the differentially expressed transcripts within the dataset, we used Cuffdiff, a separate program included in Cufflinks, to calculate expression in two or more samples and test the statistical significance of each observed change in expression between them. Cuffdiff reports numerous output files containing the results of the differential analysis of the samples, including genes and transcripts expression level changes, familiar statistics such as $\log _{2}$-fold change, $P$ values (both raw and corrected for multiple testing), and gene-related attributes such as common name and genome location. The differentially expressed genes were clustered and visualized by TreeView [14]. Functional annotations of these genes were performed by the DAVID bioinformatics resources [15] and WEB-based GEne SeT AnaLysis Toolkit (WebGestalt) [16]. Downstream enrichment analyses such as TF binding sites in promoter regions and microRNA sites in $3^{\prime}$-UTRs were performed using Expander (version 6.0.5) [17], and the miRNA-target gene network was constructed by Cytoscape [18].

2.7. $q P C R$. qPCR analyses were performed to validate the results of RNA-seq. The reverse transcription is synthesized using RevertAidTM First Strand cDNA Synthesis Kit from Fermentas according to the manufacturer's instructions. The PCR primers were designed with Primer Premier 5.0 software and $\beta$-Actin was used as a reference gene. The primer sequences, melting temperatures, and product sizes are shown in Table 1. qPCR was performed on iQ5 Real Time PCR Detection System (Bio-Rad) (Bio-Rad, USA) using SYBR Green Realtime PCR Master Mix (TOYOBO CO., LTD, Japan) as the readout. Three independent biological replicates of the control and cadmium treated groups were included in the analysis and all reactions were carried out in triplicates. Data was analyzed by the 2- $\Delta \Delta \mathrm{CT}$ method [19].

2.8. Statistical Analysis. Besides those statistical tools embedded in the bioinformatics software and resources, additional statistical analyses were performed using GraphPad Prism
(Version 5.00). Cadmium treated groups were compared with the control groups by unpaired Student's $t$-test. $P<0.05$ was considered statistically significant.

\section{Results}

3.1. Cadmium Accumulated in Mouse and Inhibited the Testosterone. After six-month cadmium-exposure treatment, all animals survived with the slight loss of body weight for the treated groups (Figure 1(a)) and did not show abnormalities or other health problems. We first tested the concentration of cadmium in serum and testis for each of the groups. As expected, cadmium concentration was significantly increased in proportion to the exposure level both in serum and testis (Figures 1(b)-1(c)). We examined the level of serum testosterone, which is essential for normal spermatogenesis and other reproductive processes. Results showed that cadmium exposure significantly reduced the level of serum testosterone in high dose cadmium treated mouse compared with the low and control mouse (Figure 1(d)).

3.2. The Next-Generation Transcriptome Sequencing. To determine the molecular events during cadmium exposure, we performed RNA-seq on testis samples of treated and untreated mice. Raw data of 80 million, 36-bp pair-ends reads were obtained and aligned to the mouse reference genome by TopHat and RNASEQR software [12,13], resulting in, on average, $71 \%$ of the reads mapped to the reference genome with $57 \%$ unique mapped reads. Then, both the unique and multiple mapped reads were kept as Cufflinks can handle the multimapped reads by uniformly dividing each multimapped read to all of the positions it maps to and calculating initial abundance. Then the mapped reads were assembled for reconstructing transcriptome and estimating expression abundance by Cufflinks. The results of estimated normalized expression levels were reported as FPKM (Fragments Per Kilobase of exon per Million fragments mapped). Overall, a total of 27600 transcripts on average were successfully reconstructed from each 




(a)

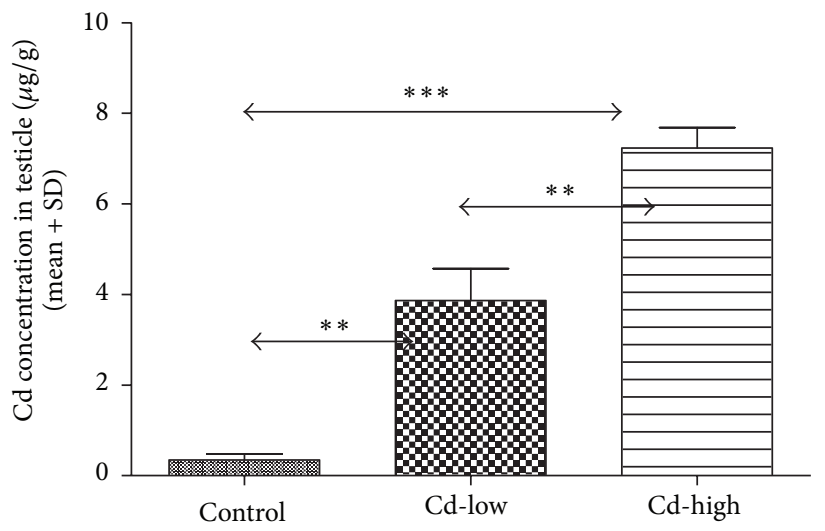

(c)



(b)

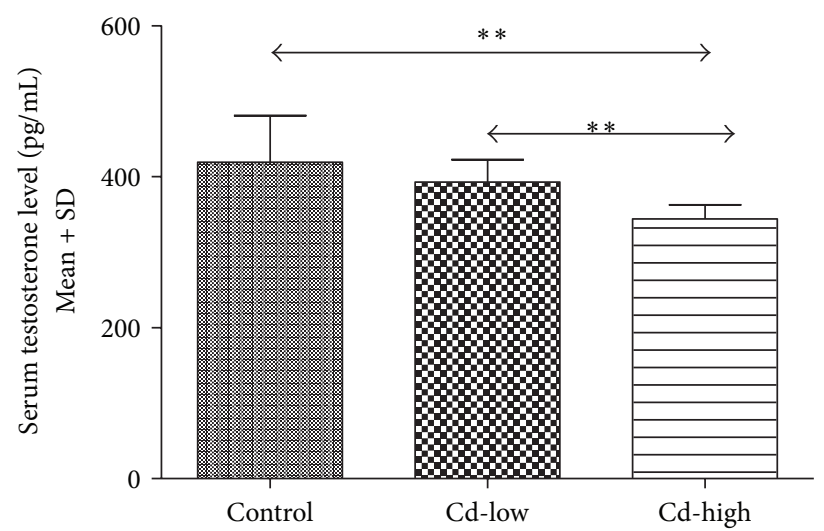

(d)

Figure 1: Body weights, level of cadmium in serum and testis, and serum testosterone. Mice were treated with different level of $\mathrm{CdCl}_{2}$ in their rodent chow for six months. (a) Body weights were measured per week during cadmium treatment. The level of accumulated cadmium in serum (b) and testis (c) were measured using the graphite furnace atomic absorption spectrometry (GFAAS) method. The levels of serum testosterone $(\mathrm{d})$ were measured by ELISA. Data was shown as mean \pm standard deviation $(n=5) .\left({ }^{* *} P<0.01,{ }^{* * *} P<0.001\right.$, Student's $t$-test).

group and mapped to the annotated genomic loci. For example, the genes on chr11:69,594,778-70,305,335 were reconstructed (Figure 2(a)) and the intergenic transcribed regions were pervasively detected compared with the mouse gene reference annotations (Figure 2(b)). Figure 2(c) shows a representative example histogram of read coverage versus a genomic loci containing the adam 24 gene.

3.3. Differentially Expressed Genes Analysis. After reads mapping, transcripts assembling, and expression level calculating, we next sought to identify differentially expressed genes between samples with different treatment. By using Cuffdiff, two expression profiles were obtained from different mapping software. We then compared them and combined the overlapped differentially expressed genes. Genes with $q$ value $<$ 0.05 were considered to be differentially expressed. Finally, a total of 830 genes were identified as differentially expressed
(Table S1 available online at http://dx.doi.org/10.1155/2014/ 529271). In detail, there were 283 differentially expressed genes between high and low groups (103 upregulated and 180 downregulated), 401 genes between high and control groups (137 upregulated and 264 downregulated), and 145 genes between low and control groups (61 upregulated and 84 downregulated), respectively (Figure 3(a)). All the differentially expressed transcripts were hierarchically clustered and the results showed that the distinct gene expression pattern was associated with cadmium exposure level, although the low and control groups exhibited a more similar expression pattern than the high group, probably because we used quite low dose of cadmium to treat the low group (Figure 3(b)).

In order to gain a comprehensive impact assessment of cadmium exposure on testicular gene expression, all biochemical pathways that altered in response to cadmium exposure were identified by comparing the ontology of all the 


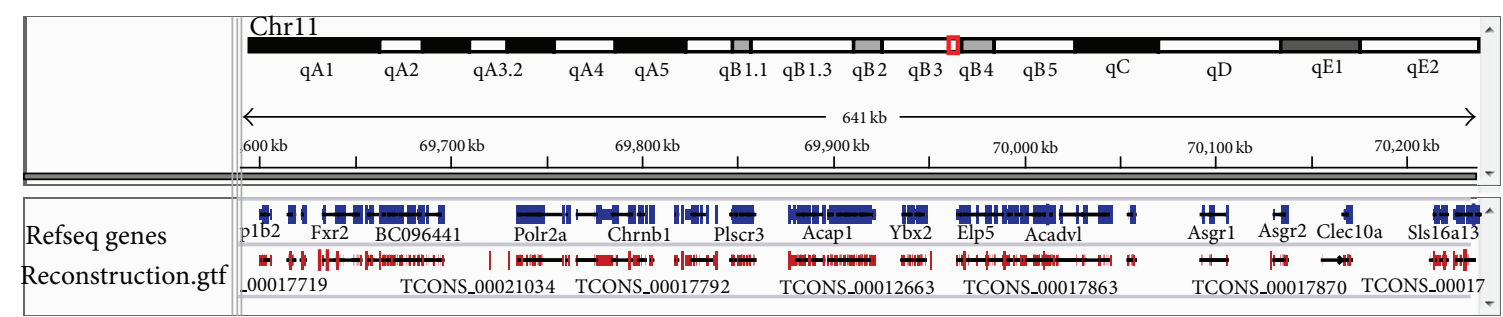

(a)

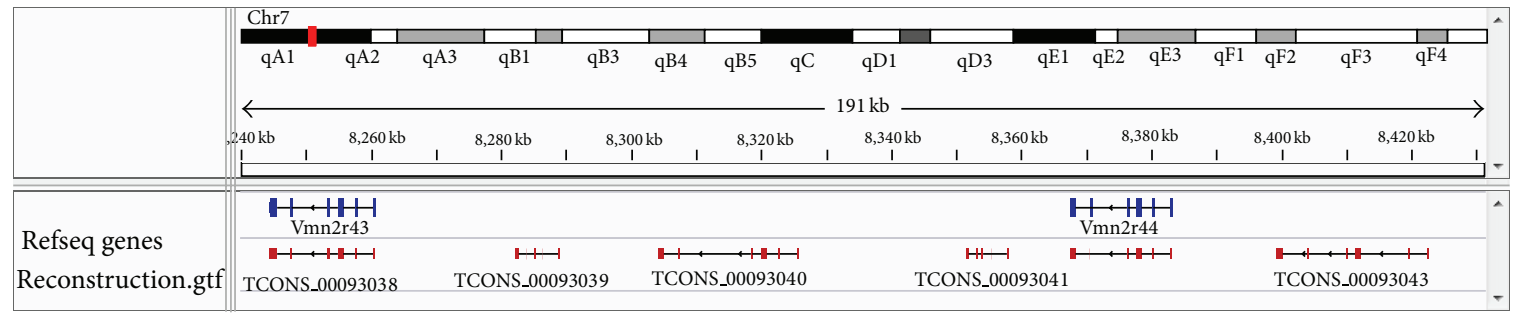

(b)



(c)

FiguRE 2: Testicular transcriptome reconstruction of RNA-seq under mouse reference annotation. (a) The genes on chr11:69,594,77870,305,335 were reconstructed as examples. (b) The intergenic transcription was detected beyond the reference annotation. (c) Read coverage of Adam24 gene on chr8 was shown.

genes differentially expressed between samples. Here, 373 differentially expressed genes annotated by UCSC and Ensembl of the 830 genes were performed with gene ontology enrichment analysis and functional classification. These genes were classified into several ontology categories according to their function in various biological processes (Figure 3(c)). Consistent with previous reports, some ontology categories that are implicated in cadmium toxicity to testis were confirmed in our study, such as genes involved in immunity, cell cycle, toxin, oxidation reduction, and metabolism [7].

Notably, the most enriched ontology category contains the genes associated with regulation of transcription. Genes that are involved in many classical signaling transduction pathways are modulated, such as Nfat5, E2f2, Fos, Junb, Notch1, and Stat4. In addition, we observed that abnormal epigenetic regulation occurred during cadmium exposure. Some of the differentially expressed genes involved in DNA methylation and histone modification are those with DNA methyltransferase, histone methyltransferase, acetyltransferase, or deacetylase activities, including Crebbp, Dmap1, Prdm9, Setd2, Prmt7, and Hdac2. Thus, both the transcriptional program and epigenetic patterns are supposed to be misregulated and implicated in cadmium caused reproductive toxicity.

Importantly, we also identified several novel and specific pathways modulated by cadmium exposure, including homeostasis of hormone (Table 2), gamete generation, and sexual reproduction (Table 3 ). Among these pathways, we noticed that similar functional categories shared the same differentially expressed candidate genes between them. 


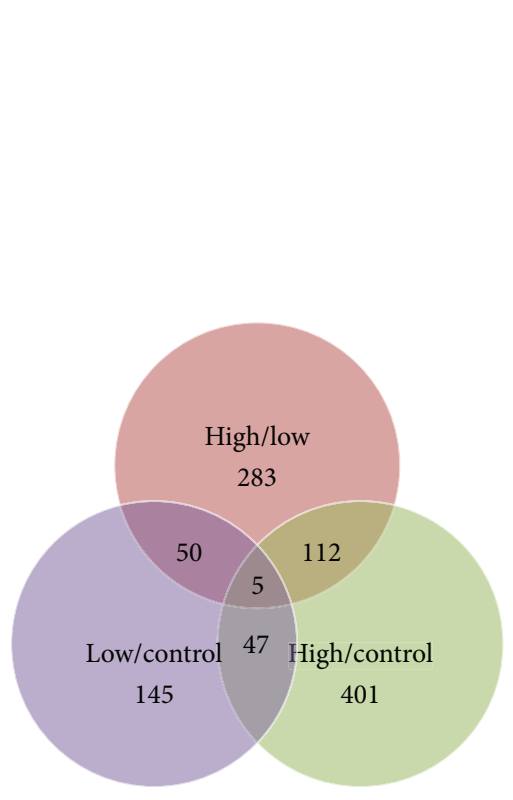

(a)

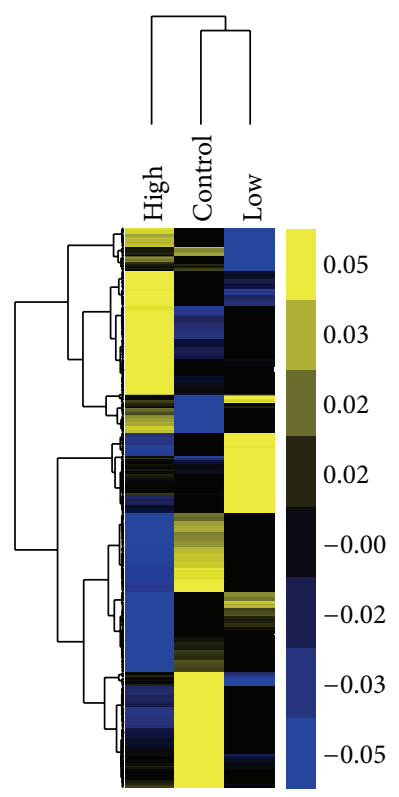

(b)

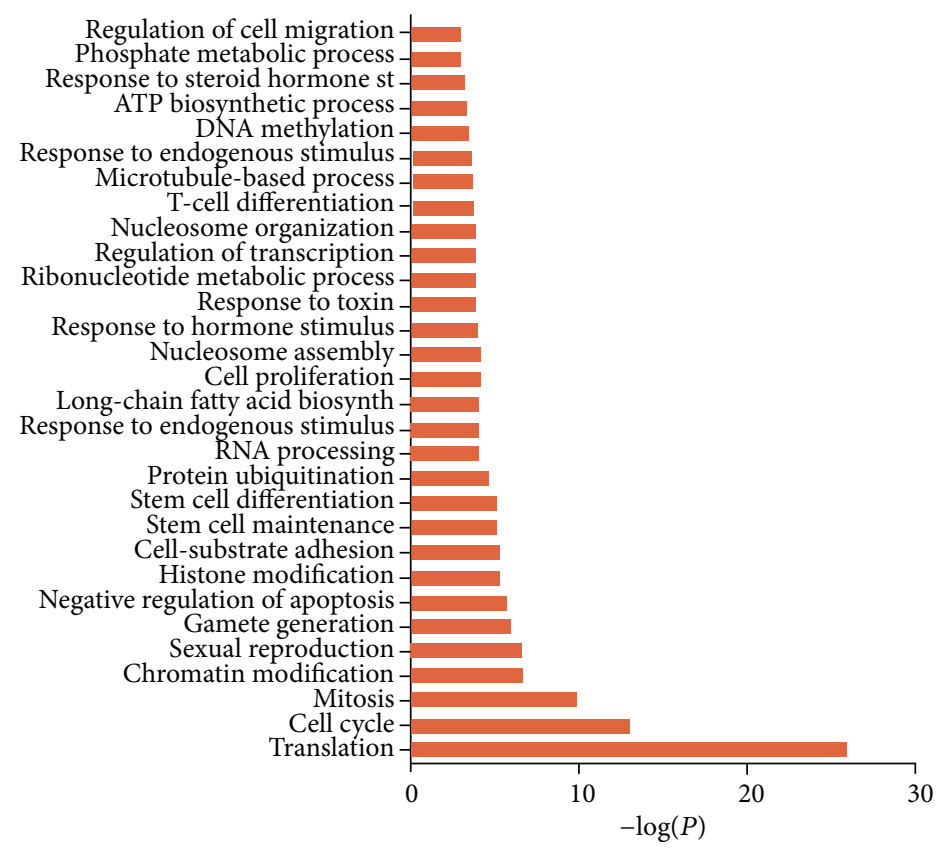

(c)

Figure 3: Cadmium modulated differentially expressed genes analysis. (a) The number of genes differentially expressed between the high level cadmium treated, low level cadmium treated, and control groups. (b) Hierarchical clustering analysis of gene expression profiles. Each column represents one mouse, and each horizontal line refers to a gene. Color legend is on the top-left of the figure. Yellow indicates genes with a greater expression relative to the geometrical means; blue indicates genes with a lower expression relative to the geometrical means. (c) Biological process Gene Ontology (GO) analysis of differentially expressed genes.

The reproduction associated functional categories comprise 16 annotated genes, most of which were inhibited due to cadmium exposure. For example, Fndc3a, Dazl, Kitl, Tex15, and $\mathrm{Zfx}$ were downregulated with the fold changes ranging from -2.6 to -4.5 . Since these genes are critical for spermatogenesis, germ cell development, or junctions between Sertoli cells [20-26], we speculated that cadmium induced testicular toxicity through targeting and downregulating these genes. The hormone related categories contained six genes, half of which were induced. In particular, the rest of three downregulated genes, named Star, Cypllal, and Cyp17al, were specifically responsible for testosterone synthetic. The downregulation of these genes may contribute to the reduction of serum testosterone in response to cadmium exposure. Collectively, these findings suggested that cadmium impaired the reproductive process and spermatogenesisand also potentially modulated 
TABLE 2: Regulation of hormone level related genes.

\begin{tabular}{llcc}
\hline Gene symbol & Description & Fold change & $Q$ value \\
\hline Adh1 & Alcohol dehydrogenase 1 (class I) & 3.806345 & 0.005 \\
Cyp1la1 & Cytochrome P450, family 11, subfamily a, polypeptide 1 & -7.197441 & $5.57 E-08$ \\
Cyp17al & Cytochrome P450, family 17, subfamily a, polypeptide 1 & -4.438219 & 0.0001 \\
Ren1, Ren2 & Renin 1 structural; similar to renin 2 tandem duplication of Ren1; & 7.678866 & 0.016 \\
Retsat & renin 2 tandem duplication of Ren1 & 4.603697 & 0.025 \\
Star & Retinol saturase (all trans retinol 13, 14 reductase) & -5.377734 & $5.57 E-05$ \\
\hline
\end{tabular}

TABLE 3: Regulation of reproductive process related genes.

\begin{tabular}{llcc}
\hline Gene symbol & Description & Fold change & Q value \\
\hline Adam24 & A disintegrin and metallopeptidase domain 24 (testase 1) & -2.872645 & 0.038 \\
Adam25 & A disintegrin and metallopeptidase domain 25 (testase 2) & -2.736529 & 0.048 \\
Adam26a & A disintegrin and metallopeptidase domain 26A (testase 3) & -3.429332 & -3.38705 \\
Dazl & Deleted in azoospermia-like & -2.636543 & 0.023 \\
Fndc3a & Fibronectin type III domain containing 3A & -4.289398 \\
Kitl & Kit ligand & 1.169034 \\
Prm2 & Protamine 2 & -3.974115 \\
Qk & Similar to Quaking protein; quaking & -2.977031 \\
Sycp2 & Synaptonemal complex protein 2 & -2.926283 \\
Tex15 & Testis expressed gene 15 & -2.973916 \\
Txndc3 & Thioredoxin domain containing 3 (spermatozoa) & -6.408031 \\
Zbtb16 & Zinc finger and BTB domain containing 16 & -3.430625 \\
Zfp37 & Zinc finger protein 37 & -4.58343 & 0.044 \\
Zfx & Zinc finger protein X-linked; similar to zinc finger protein & 0.006 \\
Zan & Zonadhesin & -3.605458 \\
\hline
\end{tabular}

the normal hormone levels during the toxic response to cadmium in testis.

Besides, 9 KEGG signaling pathways were affected by cadmium exposure by mapping the differentially expressed genes to the KEGG database. Those modulated signaling pathways were comprised of ribosome, Alzheimer's disease, asthma, oxidative phosphorylation, focal adhesion, ECM-receptor interaction, C21-Steroid hormone metabolism, metabolic pathways, and prostate cancer (Table 4). Among these overrepresented pathways, according to functional hierarchies in KEGG, pathways of asthma are associated with the immune system. ECM-receptor interaction corresponds tosignaling molecules and interaction. Focal adhesion and ribosome are associated with cell communication and translation, respectively. Three modulated pathways, C21-Steroid hormone metabolism, oxidative phosphorylation, and metabolic pathways, are associated with metabolism, indicating the basal metabolism of cells in testis are affected. Altogether, our results reflected that multiple cellular and molecular mechanismsare modulated during cadmium exposure.

3.4. Validation of RNA-Seq Data by Quantitative Real Time $P C R$ ( $q P C R)$. To confirm the changes in gene expression observed by RNA-seq, we performed qPCR analysis on three reproductions (Prm2, Tex15, and Dazl), two hormones (Cyp1lal and Cyp17a1) associated with functional categories
TABLE 4: Modulated KEGG pathways.

\begin{tabular}{lcc}
\hline Pathway name & $\begin{array}{c}\text { Number of } \\
\text { genes }\end{array}$ & $\begin{array}{c}\text { Corrected } P \\
\text { value }\end{array}$ \\
\hline Ribosome & 17 & $5.54 E-12$ \\
Alzheimer's disease & 11 & 0.0246 \\
Asthma & 2 & 0.0486 \\
Oxidative phosphorylation & 9 & 0.0419 \\
Focal adhesion & 10 & $1.73 E-4$ \\
ECM-receptor interaction & 7 & $1.15 E-4$ \\
C21-steroid hormone metabolism & 2 & 0.0106 \\
Prostate cancer & 6 & 0.00868 \\
Metabolic pathways & 37 & 0.0059 \\
\hline
\end{tabular}

genes, and a randomly selected gene named Adam9. qPCR results showed that these genes are significantly differentially expressed $(P<0.05)$ and exhibited the similar expression status compared to RNA-seq and conformed that Tex15, Dazl, Cyp1lal, and Cyp17al were inhibited by cadmium treatment (Figure 4).

3.5. Transcriptional and Posttranscriptional Control of Cadmium Modulated Genes. In an effort to uncover the potential regulatory mechanism underlying the transcription of 


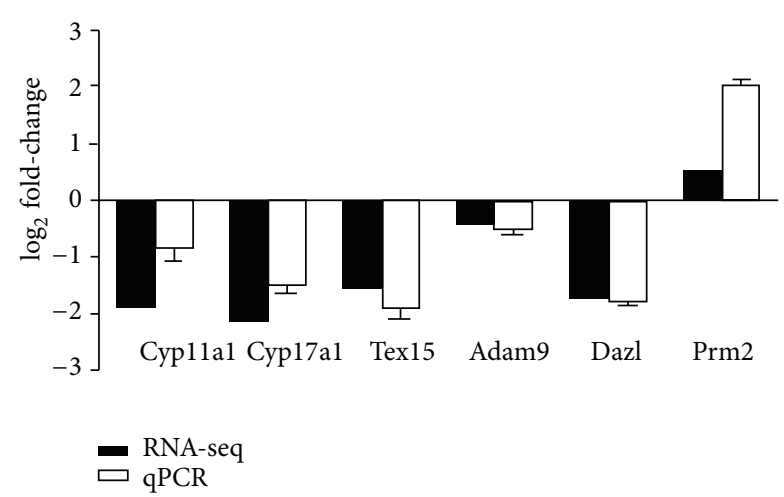

FIGURE 4: qPCR validation of the RNA-seq data. $\log _{2}$-fold change determined from the relative $\mathrm{Ct}$ values of six genes were compared to those detected by RNA-seq. Replicates $(n=3)$ of each sample were run and the $\mathrm{Ct}$ values averaged. All Ct values were normalized to beta-actin. $P$ values of the Q-PCR data are 0.002 (Cyp1lal), 0.02 (Cyp17a1), 0.012 (Tex15), 0.014 (GLRX2), 0.008 (Adam9), 0.016 (Dazl), and 0.014 (Prm2), respectively.

TABLE 5: Enrichment of transcription factors across the promoter regions of differentially expressed genes.

\begin{tabular}{lcc}
\hline Transcriptional factors & Number of genes & Corrected $P$ value \\
\hline ETF & 193 & $4.37 E-16$ \\
NKX3A & 178 & 0.007 \\
Nrf-1 & 170 & $6.53 E-4$ \\
HMGIY & 140 & 0.009 \\
SRY & 328 & $3.49 E-4$ \\
ZF5 & 199 & $2.05 E-7$ \\
FOXJ2 & 153 & $7.62 E-5$ \\
OCT-1 & 229 & $1.12 E-4$ \\
E2F-1 & 226 & $2.24 E-7$ \\
LUN-1 & 35 & 0.043 \\
FOXP1 & 319 & $7.31 E-7$ \\
AP2 & 169 & 0.014 \\
\hline
\end{tabular}

the cadmium modulated gene sets, we performed transcription factor binding sites analysis within the promoters and microRNA targets analysis of the cadmium modulated genes. Promoter regions for positions of $-1000-+200$ of the TSS across the cadmium modulated genes were predicted for the binding sites enrichment of several transcription factors $(P<$ 0.05 , Bonferroni test) (Table 5). Gene ontology analysis of these transcription factors revealed that they were involved in multiple biological processes containing regulation of cell cycle, hormone secretion, organ morphogenesis, reproductive process, neurogenesis, and response to stimulus, which were in accordance with the biological processes associated with the differentially expressed genes regulated by these transcription factors (Figure 5(a)).

We next performed microRNA targets analysis of the differentially expressed genes for further investigating the posttranscriptional control of them. A total of 10 microRNAs were identified as significantly enriched at $3^{\prime}$-UTR region of the differentially expressed genes (FDR $<0.05)$ (Table 6). By
TABLE 6: MicroRNAs enriched at 3 '-UTR region of the differentially expressed genes.

\begin{tabular}{lcc}
\hline MicroRNA & $\begin{array}{c}\text { Number of } \\
\text { targeted genes }\end{array}$ & FDR \\
\hline Mir-142-3p & 6 & 0.001 \\
Mir-342/342-3p & 5 & 0.0015 \\
Mir-196ab & 5 & 0.0025 \\
Mir-874 & 4 & 0.003 \\
Mir-124/506 & 5 & 0.0135 \\
Mir-30a & 3 & 0.0075 \\
Mir-124/506 & 11 & 0.0205 \\
Mir-153 & 4 & 0.0265 \\
Mir-25/32/92/92ab/363/367 & 3 & 0.0195 \\
Mir-448 & 4 & 0.006 \\
\hline
\end{tabular}

evaluating the microRNAs-Target-Network generated from the above information, it is implicated that a number of altered genes expression in this study may be regulated by the common microRNAs (Figure 5(b)), indicating their potential roles in regulating the reproductive process in response to cadmium exposure.

\section{Discussion}

Cadmium has been suggested to be anenvironmental and occupational toxic heavy metal that causes several diseases and toxically targets the lung, the liver, the kidney, the bone, the cardiovascular system, the immune system, and the reproductive system [2]. Here, in order to uncover the exclusive molecular mechanism of the mouse reproductive toxicity caused by chronic cadmium exposure, we simulated the conditions of cadmium pollution in human by treating the mouse with different doses of cadmium for 6 months. We observed cadmium exposure significantly reduced the level of serum testosterone. As a member of androgens, testosterone brings about its biological functions through associations with androgen receptor (AR), leading to AR transactivation which then results in the modulation of AR downstream gene expressions [27]. While the difference of testosterone seems to be small, such difference may lead to significantly downstream effects through the cascade signal transduction and transcriptional regulation of many genes by androgen receptor.

We next used RNA-seq to analyze the transcriptome of mouse testis affected by cadmium. We found a total of 830 genes and transcripts that were differentially expressed. Gene Ontology analysis revealed that these genes were enriched in several biological processes, in which the genes related to the reproductive process were paid more attention. For example, Fndc3a was reported to be required for adhesion between spermatids and Sertoli cells during mouse spermatogenesis [20]. Loss of Tex15 function causes early meiotic arrest in male mice, and Tex15-deficient spermatocytes exhibit a failure in chromosomal synapsis and DNA double-strand breaks are impaired [21]. In human, the deletion of DAZ 


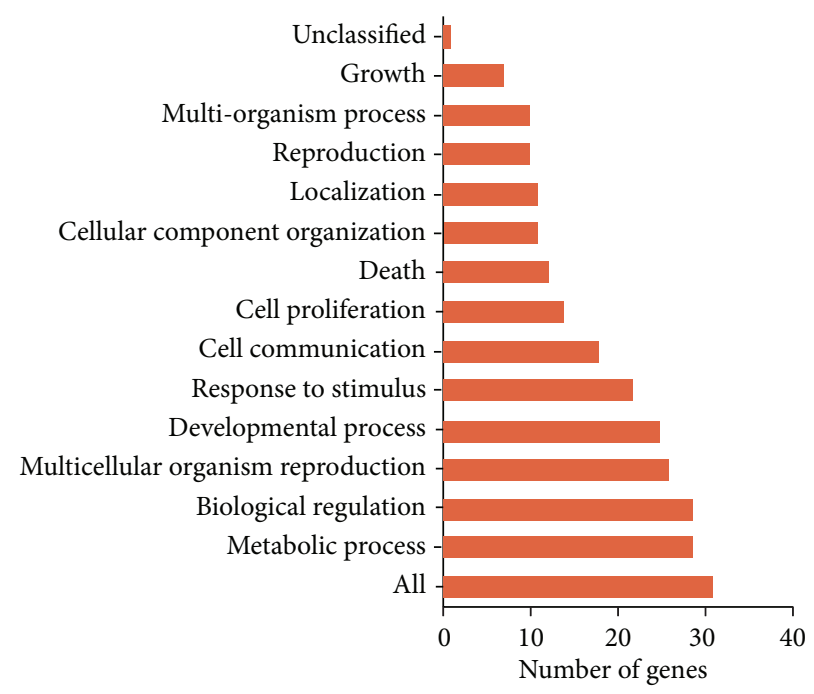

(a)

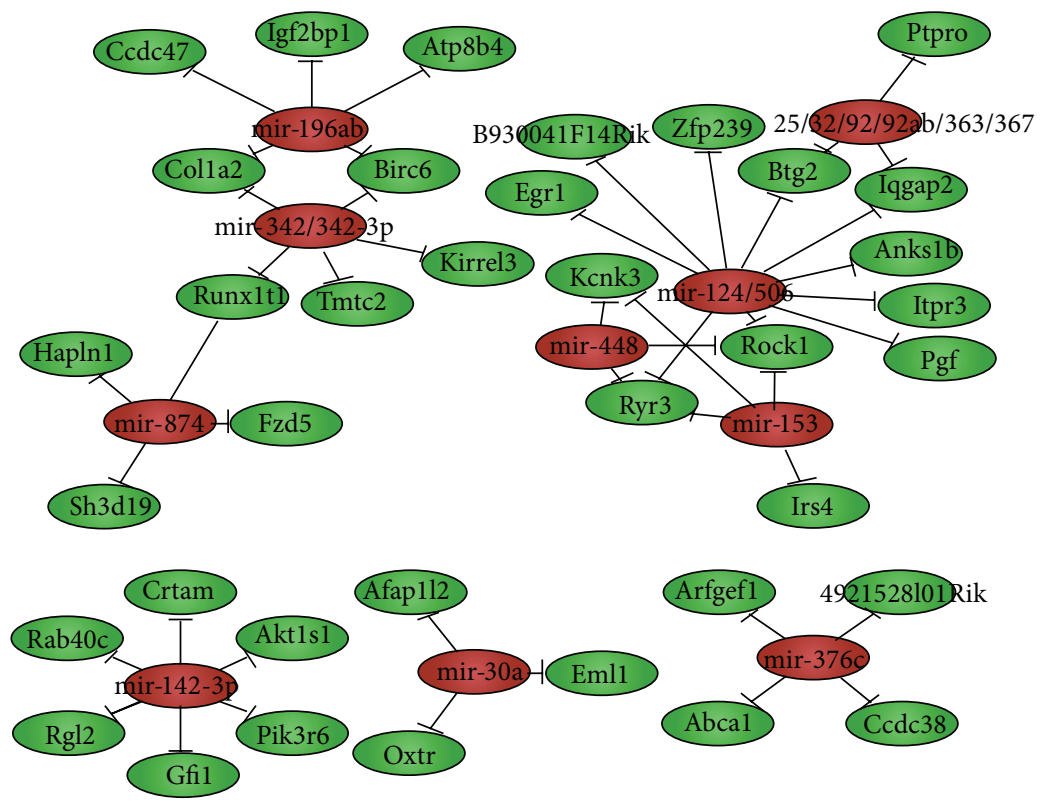

(b)

FIGURE 5: Transcriptional regulation analysis of the differentially expressed genes. (a) Biological process gene ontology analysis of the transcription factors that regulate the gene expression. (b) MicroRNA-target gene network. Red circles represent microRNAs; green circles represent the target genes.

cluster is associated with azoospermia and oligospermia in $5-10 \%$ of infertile men [22], and disruption of the Dazla gene leads to loss of germ cells and complete absence of gamete production [23]. In mouse, Dazl is required for embryonic development and survival of XY germ cells [24]. Kitl mutant mice exhibited reduced testis size due to aberrant spermatogonial proliferation, affecting the formation of tight junctions between Sertoli cells during postnatal development [25]. The zinc-finger proteins ZFX mutant mice were smaller and less viable and had fewer germ cells than wild-type mice [26]. Altogether, we supposed that the cadmium could cause the impairment of spermatogenesis and testicular toxicity through the repression of these genes. In addition, we identified six hormone related genes that were modulated by cadmium. We observed the decrease of expression for Star, Cyp1la1, and Cyp17al, which were in accordance with a previous study [28]. As these genes encode the primarily testosterone synthetic enzymes, it is likely that the cadmium perturbed the spermatogenesis through repressing the synthesis of testicular testosterone as well. Combined with other modulated functional categories such as immunity, cell cycle, transcription, epigenetic regulation, and metabolism, the molecular mechanisms of cadmium caused male reproductive toxicity are implicated in multiple layers of deregulation of several biological processes.

Further, we computationally analyzed the transcriptional and posttranscriptional control of the differentially expressed genes. We found several transcriptional factors were enriched 
with the binding sites at the promoter regions of some gene sets. These binding events should be verified by further ChIP experiments. While these transcriptional factors were unable to be detected as statistically significantly differentially expressed between the samples, it is likely that the slight change of expression ultimately led to the significant expression change of their targets. We also predicted the microRNAs with the binding possibility of some sets of cadmium modulated genes. We identified 10 microRNAs targeted to the differentially expressed genes, the regulatory roles of which in testis response to cadmium could be explored by their expression patterns and the gain- or loss-of-function studies in the future.

In summary, our study demonstrated that many genes in testis were modulated due to chronic cadmium exposure. In particular, aside from the genes related to the functional categories previously reported, we identified novel pathways and the potential transcriptional regulatory mechanism on the cadmium modulated genes. These findings provide evidence for the elucidation of the molecular mechanism linking the chronic cadmium exposure to the impairment of male reproductive system and the clues for future studies of potential biomarkers and therapeutic targets for cadmium exposure.

\section{Conflict of Interests}

The authors declare no conflict of interests.

\section{Acknowledgments}

The authors thank Jie Li at the Department of Pharmacology, Basic Medical School of Wuhan University for technical help. They also thank all members in Dr. Zhong's laboratory for helpful suggestions and technical assistance. This work was financially supported by the National Natural Science Foundation of China (Grant no. 31172395) and Key Technologies Research and Development Program of China (Grant no. 2013BAI12B01-3).

\section{References}

[1] S. Satarug and M. R. Moore, "Adverse health effects of chronic exposure to low-level cadmium in foodstuffs and cigarette smoke," Environmental Health Perspectives, vol. 112, no. 10, pp. 1099-1103, 2004.

[2] B. A. Fowler, "Monitoring of human populations for early markers of cadmium toxicity: a review," Toxicology and Applied Pharmacology, vol. 238, no. 3, pp. 294-300, 2009.

[3] M. Waisberg, P. Joseph, B. Hale, and D. Beyersmann, "Molecular and cellular mechanisms of cadmium carcinogenesis," Toxicology, vol. 192, no. 2-3, pp. 95-117, 2003.

[4] Y. H. Jin, A. B. Clark, R. J. C. Slebos et al., "Cadmium is a mutagen that acts by inhibiting mismatch repair," Nature Genetics, vol. 34, no. 3, pp. 326-329, 2003.

[5] B. Wang, Y. Li, Y. Tan et al., "Low-dose Cd induces hepatic gene hypermethylation, along with the persistent reduction of cell death and increase of cell proliferation in rats and mice," PLOS ONE, vol. 7, no. 3, Article ID e33853, 2012.
[6] O. Akinloye, A. O. Arowojolu, O. B. Shittu, and J. I. Anetor, "Cadmium toxicity: a possible cause of male infertility in Nigeria," Reproductive Biology, vol. 6, no. 1, pp. 17-30, 2006.

[7] J. Thompson and J. Bannigan, "Cadmium: toxic effects on the reproductive system and the embryo," Reproductive Toxicology, vol. 25, no. 3, pp. 304-315, 2008.

[8] A. M. Ronco, M. Montenegro, P. Castillo et al., "Maternal exposure to cadmium during gestation perturbs the vascular system of the adult rat offspring," Toxicology and Applied Pharmacology, vol. 251, no. 2, pp. 137-145, 2011.

[9] M. L. Hanson, I. Holásková, M. Elliott, K. M. Brundage, R. Schafer, and J. B. Barnett, "Prenatal cadmium exposure alters postnatal immune cell development and function," Toxicology and Applied Pharmacology, vol. 261, no. 2, pp. 196-203, 2012.

[10] S. Dakeshita, T. Kawai, H. Uemura et al., "Gene expression signatures in peripheral blood cells from Japanese women exposed to environmental cadmium," Toxicology, vol. 257, no. 1-2, pp. 25-32, 2009.

[11] G.-Y. Li, M. Kim, J.-H. Kim, M.-O. Lee, J.-H. Chung, and B.H. Lee, "Gene expression profiling in human lung fibroblast following cadmium exposure," Food and Chemical Toxicology, vol. 46, no. 3, pp. 1131-1137, 2008.

[12] C. Trapnell, A. Roberts, L. Goff et al., "Differential gene and transcript expression analysis of RNA-seq experiments with TopHat and Cufflinks," Nature Protocols, vol. 7, no. 3, pp. 562578, 2012.

[13] L. Y. Chen, K.-C. Wei, A. C.-Y. Huang et al., "RNASEQR-a streamlined and accurate RNA-seq sequence analysis program," Nucleic Acids Research, vol. 40, no. 6, article e42, 2012.

[14] M. B. Eisen, P. T. Spellman, P. O. Brown, and D. Botstein, "Cluster analysis and display of genome-wide expression patterns," Proceedings of the National Academy of Sciences of the United States of America, vol. 95, no. 25, pp. 14863-14868, 1998.

[15] D. W. Huang, B. T. Sherman, and R. A. Lempicki, "Systematic and integrative analysis of large gene lists using DAVID bioinformatics resources," Nature Protocols, vol. 4, no. 1, pp. 44-57, 2009.

[16] B. Zhang, S. Kirov, and J. Snoddy, "WebGestalt: an integrated system for exploring gene sets in various biological contexts," Nucleic Acids Research, vol. 33, no. 2, pp. W741-W748, 2005.

[17] R. Shamir, A. Maron-Katz, A. Tanay et al., "EXPANDER-an integrative program suite for microarray data analysis," $B M C$ Bioinformatics, vol. 6, article 232, 2005.

[18] M. S. Cline, M. Smoot, E. Cerami et al., "Integration of biological networks and gene expression data using Cytoscape," Nature Protocols, vol. 2, no. 10, pp. 2366-2382, 2007.

[19] K. J. Livak and T. D. Schmittgen, "Analysis of relative gene expression data using real-time quantitative PCR and the 2$\Delta \Delta$ CT method," Methods, vol. 25, no. 4, pp. 402-408, 2001.

[20] K. L. Obholz, A. Akopyan, K. G. Waymire, and G. R. MacGregor, "FNDC3A is required for adhesion between spermatids and Sertoli cells," Developmental Biology, vol. 298, no. 2, pp. 498-513, 2006.

[21] F. Yang, S. Eckardt, N. A. Leu, K. J. McLaughlin, and P. J. Wang, "Mouse TEX15 is essential for DNA double-strand break repair and chromosomal synapsis during male meiosis," Journal of Cell Biology, vol. 180, no. 4, pp. 673-679, 2008.

[22] R. Reijo, T.-Y. Lee, P. Salo et al., "Diverse spermatogenic defects in humans caused by $\mathrm{Y}$ chromosome deletions encompassing a novel RNA-binding protein gene," Nature Genetics, vol. 10, no. 4, pp. 383-393, 1995. 
[23] M. Ruggiu, R. Speed, M. Taggart et al., "The mouse Dazla gene encodes a cytoplasmic protein essential for gametogenesis," Nature, vol. 389, no. 6646, pp. 73-77, 1997.

[24] Y. Lin and D. C. Page, "Dazl deficiency leads to embryonic arrest of germ cell development in XY C57BL/6 mice," Developmental Biology, vol. 288, no. 2, pp. 309-316, 2005.

[25] S. Deshpande, V. Agosti, K. Manova, M. A. S. Moore, M. P. Hardy, and P. Besmer, "Kit ligand cytoplasmic domain is essential for basolateral sorting in vivo and has roles in spermatogenesis and hematopoiesis," Developmental Biology, vol. 337, no. 2, pp. 199-210, 2010.

[26] S.-W. Luoh, P. A. Bain, R. D. Polakiewicz et al., "Zfx mutation results in small animal size and reduced germ cell number in male and female mice," Development, vol. 124, no. 11, pp. 22752284, 1997.

[27] H. V. Heemers and D. J. Tindall, "Androgen receptor (AR) coregulators: a diversity of functions converging on and regulating the AR transcriptional complex," Endocrine Reviews, vol. 28, no. 7, pp. 778-808, 2007.

[28] Y.-L. Ji, H. Wang, P. Liu et al., "Pubertal cadmium exposure impairs testicular development and spermatogenesis via disrupting testicular testosterone synthesis in adult mice," Reproductive Toxicology, vol. 29, no. 2, pp. 176-183, 2010. 

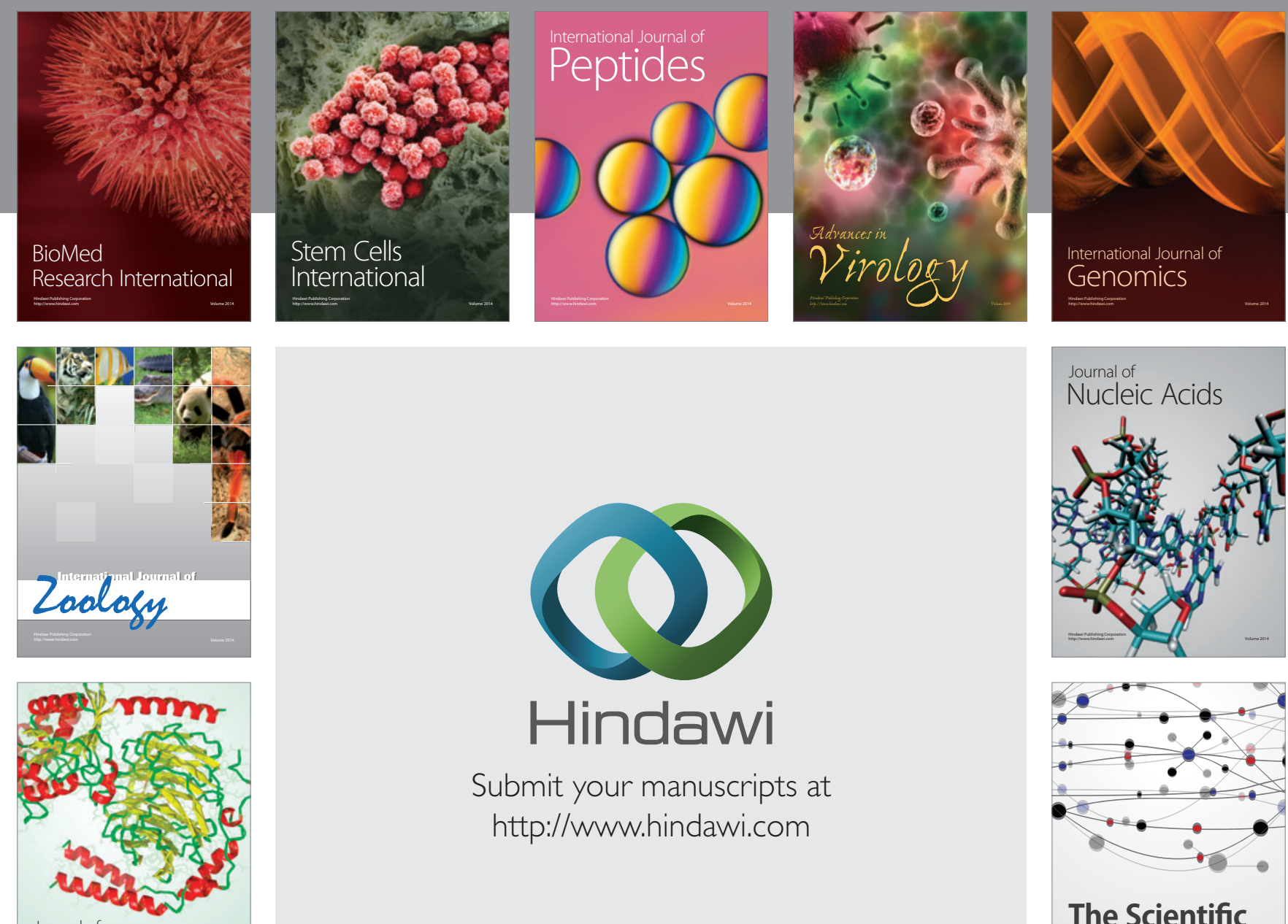

Submit your manuscripts at

http://www.hindawi.com

Journal of
Signal Transduction


The Scientific World Journal
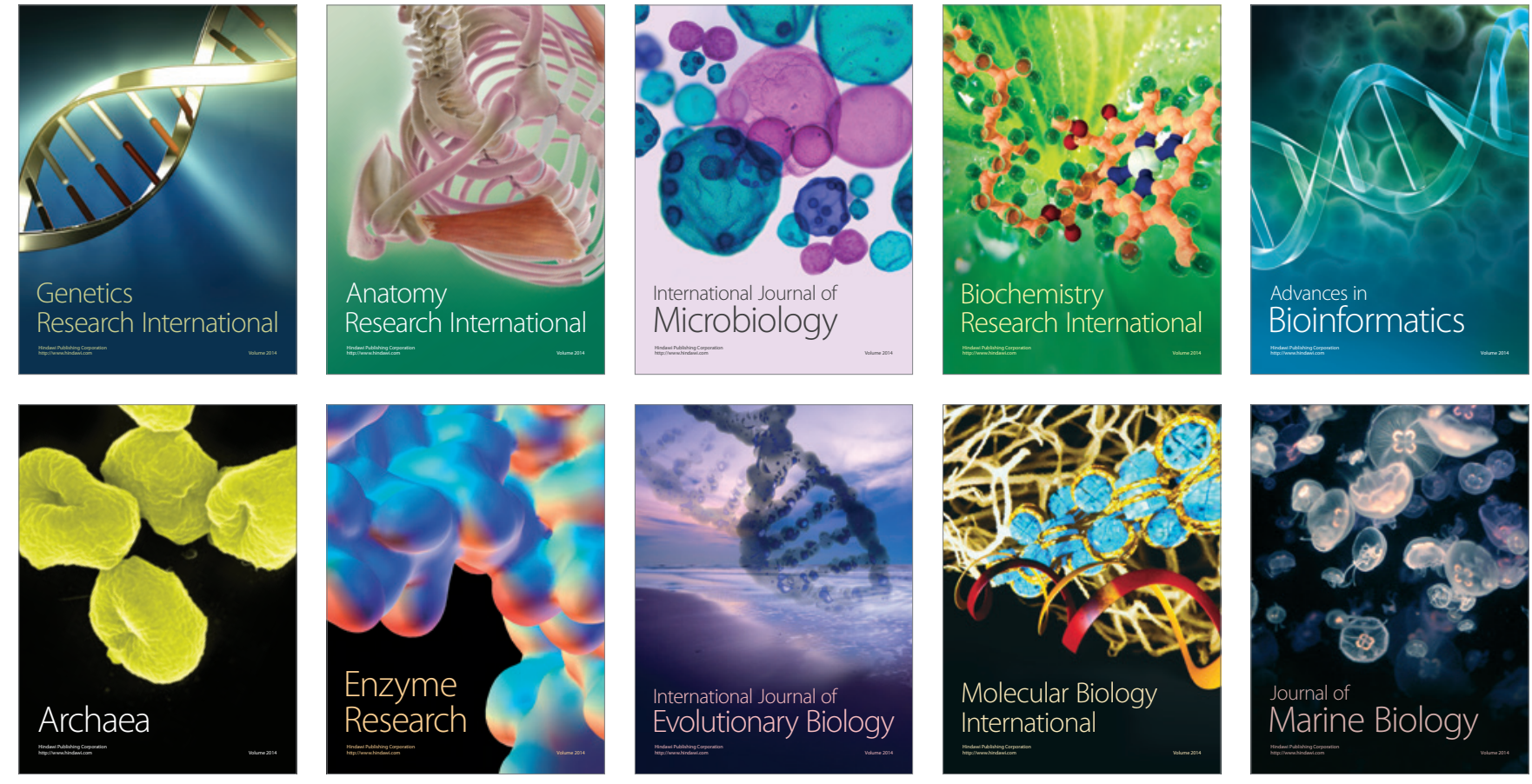